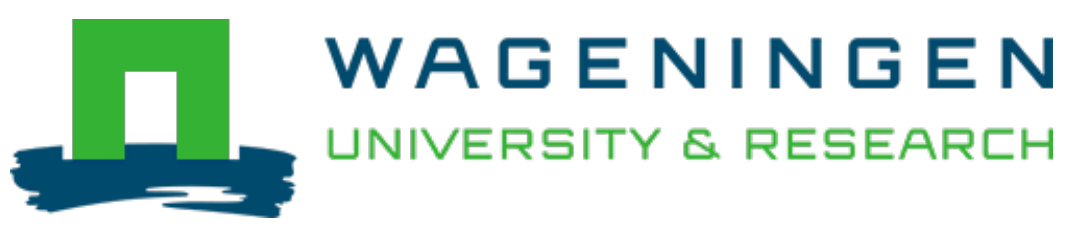

\title{
Improving data standards and integration for more effective decision-making in agriculture
}

Improving data management and decision support systems in agriculture

Wolfert, J.

https://doi.org/10.19103/as.2020.0069.03

This article is made publicly available in the institutional repository of Wageningen University and Research, under the terms of article $25 \mathrm{fa}$ of the Dutch Copyright Act, also known as the Amendment Taverne. This has been done with explicit consent by the author.

Article 25 fa states that the author of a short scientific work funded either wholly or partially by Dutch public funds is entitled to make that work publicly available for no consideration following a reasonable period of time after the work was first published, provided that clear reference is made to the source of the first publication of the work.

This publication is distributed under The Association of Universities in the Netherlands (VSNU) 'Article $25 \mathrm{fa}$ implementation' project. In this project research outputs of researchers employed by Dutch Universities that comply with the legal requirements of Article $25 \mathrm{fa}$ of the Dutch Copyright Act are distributed online and free of cost or other barriers in institutional repositories. Research outputs are distributed six months after their first online publication in the original published version and with proper attribution to the source of the original publication.

You are permitted to download and use the publication for personal purposes. All rights remain with the author(s) and / or copyright owner(s) of this work. Any use of the publication or parts of it other than authorised under article $25 \mathrm{fa}$ of the Dutch Copyright act is prohibited. Wageningen University \& Research and the author(s) of this publication shall not be held responsible or liable for any damages resulting from your (re)use of this publication.

For questions regarding the public availability of this article please contact openscience.library@wur.nl 


\section{Improving data}

management and

decision support

systems in agriculture

Edited by Dr Leisa Armstrong, Edith Cowan University, Australia

\section{ECHLAPTER FRONI THIS BOOKS}

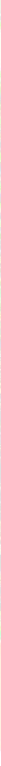




\section{Improving data standards and integration for more effective decision-making in agriculture}

Sjaak Wolfert, Wageningen University and Research, The Netherlands

1 Introduction

2 Business process modelling to identify data requirements

3 Data flows for a particular process: the example of variable rate fertilization

4 Linking platforms and software

5 Creating a reference architecture for interoperability, replicability and re-use

6 Key elements in data management

7 Conclusions

8 Where to look for further information

9 References

\section{Introduction}

As sensors and smart machines become more common on farms, and farm data grow in quantity and scope, farming processes will become increasingly datadriven and data-enabled. Rapid developments in the Internet of Things (IoT) and cloud computing enable the development of 'smart farming' (Sundmaeker et al., 2016). Precision agriculture technology has focussed on identifying in-field variability as a basis for more targeted management (e.g. more selective applications of fertilizer, pesticides or herbicides). 'Smart farming' goes beyond that by basing management tasks not only on location but also on other data, enhanced by context awareness and situation awareness, triggered by realtime events (Wolfert et al., 2014). By linking sensors, data acquisition, decision support systems and robotics, smart farming offers the possibility of agile, autonomous systems capable of predicting what is needed, making decisions and implementing them as well as responding intelligently to unforeseen events. 
Real-time reconfiguration features are required to ensure agility in the system, especially in cases of sudden changes in operational conditions (e.g. changes in weather or a disease outbreak). These features typically include intelligent assistance in the implementation, maintenance and use of the technology. Figure 1 summarizes the concept of 'smart farming' along the management cycle as a cyber-physical system. 'Smart farming' means that smart devices - connected to the Internet - control the farm system. Smart devices enhance the use of conventional tools (e.g. rain gauges, tractors, notebooks). They add autonomous context-awareness using all kinds of sensors. They also add built-in intelligence, capable of executing autonomous actions. Robots can play an increasingly important role. Where humans are still involved, their role in analysis, planning, implementation and monitoring is assisted by machines so that the cyber-physical cycle becomes almost autonomous. Humans will always be involved in the whole process, but increasingly at the level of design and monitoring, leaving most operational activities to machines.

However, the increasing number of devices and related systems needed for 'smart farming' create new challenges for the exchange of data required. There is a clear need for data standards to address this challenge. The objective of this chapter is to explain how standards for data exchange should be developed for effective decision-making in agriculture. I will argue that

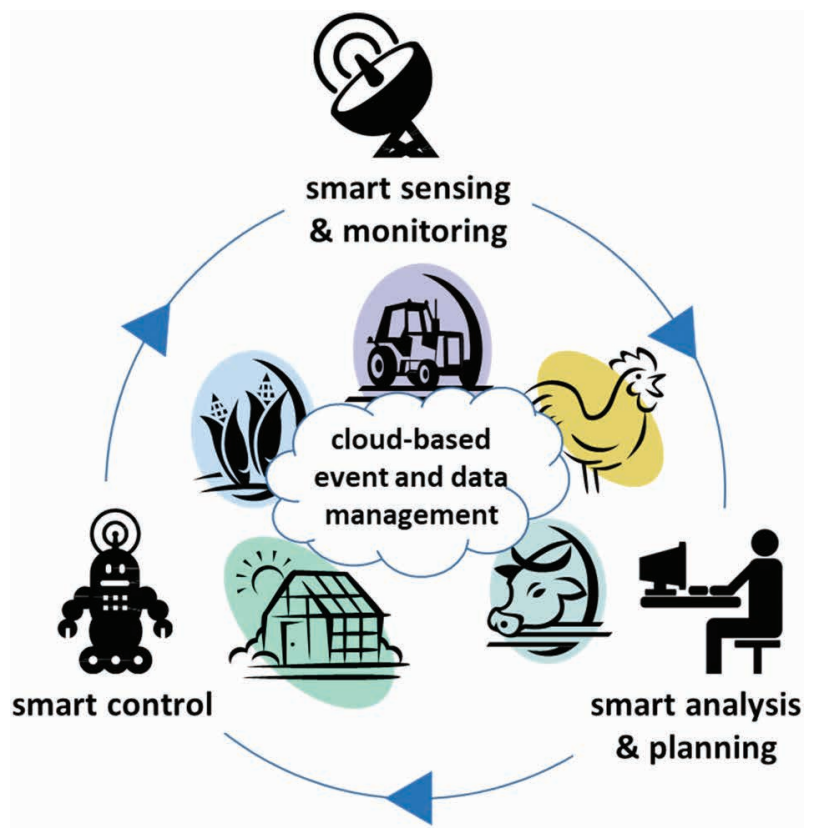

Figure 1 Three-tiered service-oriented architecture with some illustrative examples from the farming sector. Source: adapted from Wolfert et al. (2010). 
integration and standardization should not start with the databases themselves, but more from the level of business processes in which data exchange plays a role. This will be illustrated by a precision agriculture example for variable-rate fertilization. Since software increasingly is being developed as smartphone apps and incorporated into a platform, we will describe how standardization plays a role in that context. The chapter will also discuss an overarching reference architecture for interoperability, replicability and re-use that was developed in the European loF2020 project. This can function as a framework for future standardization in smart-farming applications.

\section{Business process modelling to identify data requirements}

Information and communication technology (ICT) is often considered as a specialist technical subject to be undertaken by experts such as hardware engineers or software programmers. However, these experts usually do not have a full understanding of the business for which they may be supplying technical products, including the key decision-making processes and systems within the business. This is particularly true for whole supply chain networks that involve different companies. It is very important to start from an understanding of business processes and then see what data and information these processes require, and which need to be exchanged between different systems. This is shown in Fig. 2. Software and other technicians often start at the application service layer at the bottom and see how they can connect different databases so that the data can be used by different users. In our view, it is more important to start the planning and analysis from the top layer, the business process management layer, and then see what data this suggests is needed and how this could be retrieved from the various data sources. In this way actual processes in the business will be supported by applications in a more appropriate way.

An example is a standard crop production process from sowing to harvesting. Production involves several stages at which key decisions need to be made. In the sowing process a farmer decides which crop and which variety has to be grown, on which field and so on. To make these decisions he/she has to get market information, for example, on what markets he/she is growing the crop for, what restrictions there may be in choosing which variety and so on. This information may need to be retrieved from different market information systems and data sources. It would be helpful if the farmer could get this information in a standardized and user-friendly way through one service, for example, showing up as 'get restrictions information' on what varieties he/she is permitted to plant. These data-driven, decision-making tools at the business process management layer of the system may be even more complicated when dealing with an integrated supply chain involving many stakeholders. 


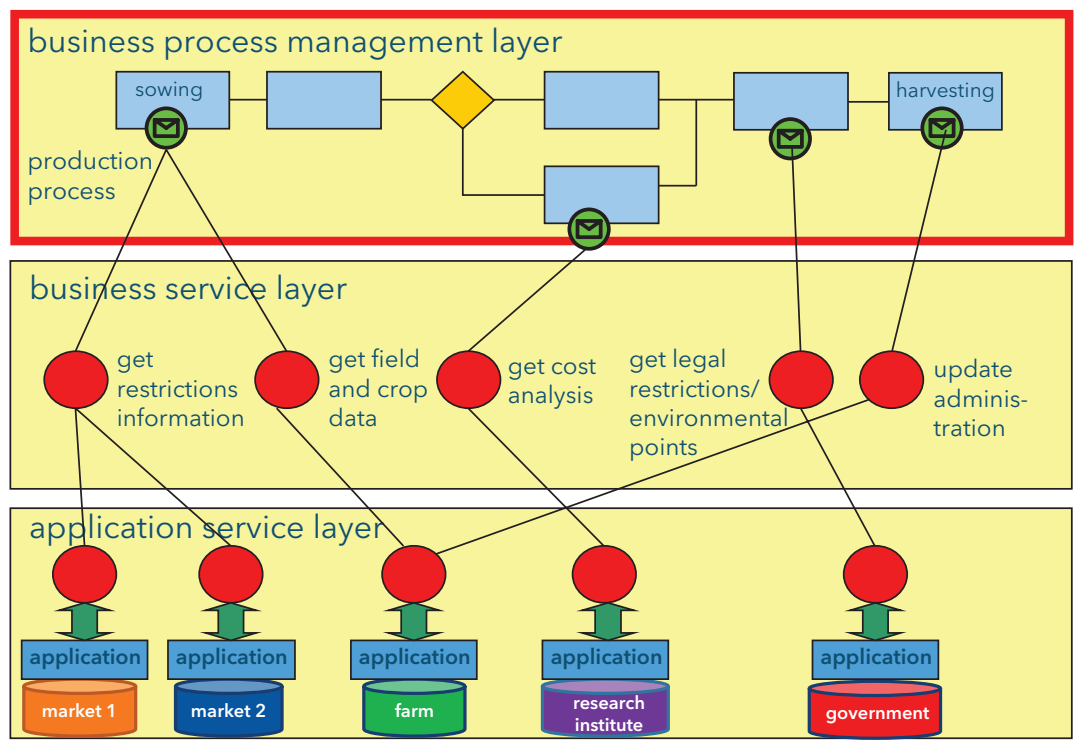

Figure 2 Three-tiered service-oriented architecture with some illustrative examples from the farming sector. Source: adapted from Wolfert et al. (2010).

Figure 2 illustrates other information flows that are needed to support the business processes in the upper layer. The envelope icons (shown in green) indicate when an information flow (from or to the process) is involved. In some cases, external information is not needed to carry out a certain process (in which case there is no envelope icon). In the next section we will illustrate information flows for a specific process: variable rate fertilization.

\section{Data flows for a particular process: the example of variable rate fertilization}

Variable rate fertilization is a precision agriculture technique in which fertilizer is applied in differing amounts in a field depending on site-specific conditions (e.g. according to the specific nutrient requirements of individual plants). To be able to decide what part of the field needs more or less fertilizers, you need site-specific information about the crop and/or the field. The process is shown in Fig. 3.

The business process starts with a recommendation request from a farm management system based on specific field and crop information. The ' $N$ advice process' ( $\mathrm{N}$ refers to nitrogen - a key crop nutrient) can be seen as the central process engine that drives the other processes. It will request data about the crop status, for example, from a leaf area index (LAI) map (which uses leaf colour to measure the nutrient status of a plant). This can be combined with 


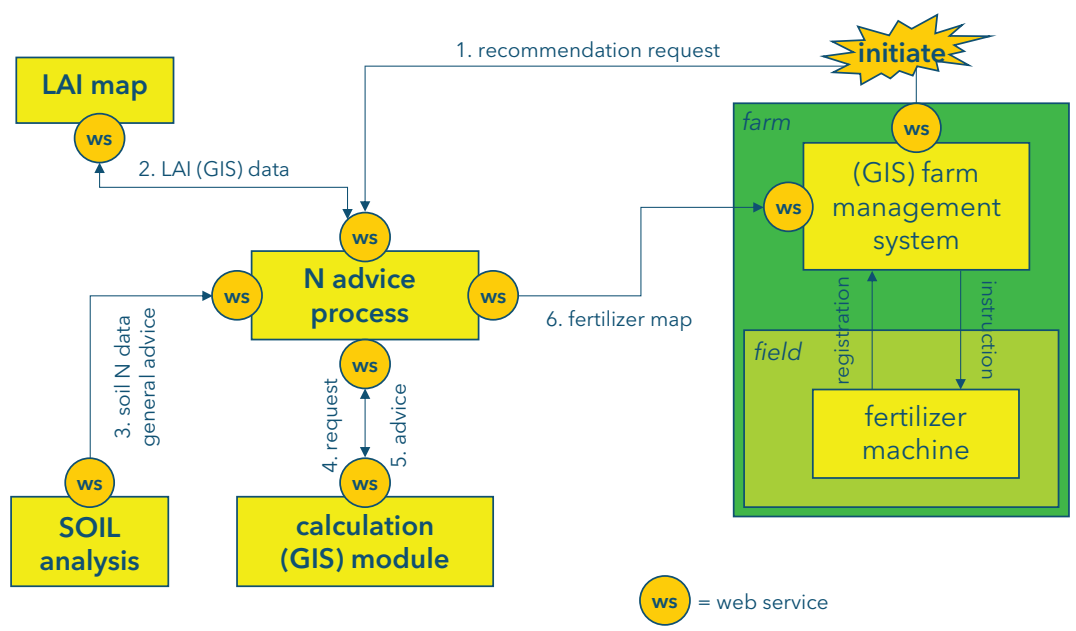

Figure 3 Conceptual graphical representation of the various data flows between different entities for variable rate fertilization. Source: adapted from Wolfert et al. (2010).

an assessment of general nitrogen (N) status for that field based on a standard soil analysis. This information can be combined with a GIS-based calculation model that identifies what fertilizer is needed at any particular point in the field. This is converted into a machine-readable fertilizer map that can be sent to the fertilizer equipment to apply the fertilizer (either directly to the machine or through the farm management system). In the past all this information was provided on paper. A web service (ws) makes it possible for data to be read and understood by different parts of the system. In practice, effective data exchange to enable the system to work is still limited by a lack of standardization in the underlying data.

Figure 3 helps to show what data have to be exchanged and which applications have to be developed. The next step is to develop a business process model as shown in Fig. 4 for the example of variable rate fertilization. The model clearly indicates the interaction between different actors or subdepartments within an organization in the so-called swim lanes. This makes it very clear on who the relevant actors are and where the information interactions take place. There are special software tools available to draw these diagrams, provide more information for each process and define the data flows. These tools make it possible to automatically generate computer code that can then be deployed as a set of web services.

\section{Linking platforms and software}

When the development of a software application is the responsibility of one software provider, standardization is not difficult because the provider can set 


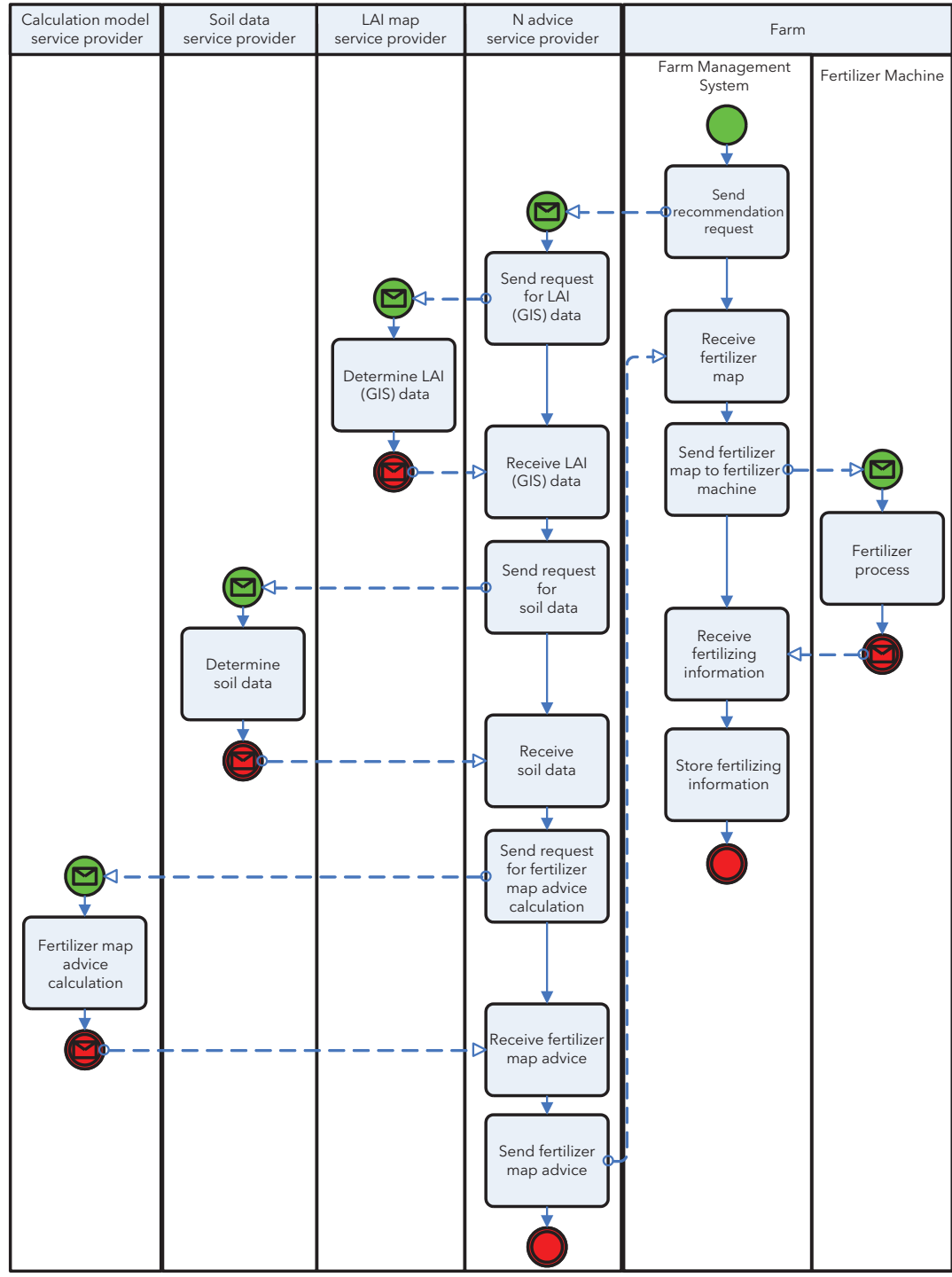

Figure 4 Business process model of the variable rate fertilizing case with various actors and subsystems in 'swimlanes'. Source: adapted from Wolfert et al. (2010).

his/her own standards. This means that every application, module and so on can easily communicate with the others. However, this is often not the case and applications are developed by different providers who may have different development methods and standards. This is especially true in agriculture and other sectors where farmers, for example, will frequently use mobile phone 'apps' which are compact, flexible and easy to use. The key challenge is how 
to make all these apps, developed from different vendors, work together seamlessly - that is, make them interoperable - in a multi-faceted and multistakeholder business process as described for variable rate fertilization.

European researchers have done significant work to develop an open infrastructure for data exchange within the Future Internet programme. This was undertaken through FIWARE, a generic open standard platform for developing open source software (www.fiware.org). A key feature of the programme was the development of a series of Generic Enablers (GEs). GEs are general purpose platform functions available through application programming interfaces (APIs). They are basic building blocks used in the development of smart 'apps' designed to have common shared functions serving multiple-use cases. GEs were developed for applications such as cloud hosting, data and context management services, loT services, security and big data analysis. The SmartAgriFood project proposed a conceptual architecture for applications for the agri-food domain based on these FIWARE GEs (Kaloxylos et al., 2012). The Flspace project implemented this architecture into a real platform for business collaboration which is shown in Fig. 5 (Verdouw et al., 2016; Wolfert et al., 2014).

FIspace uses FIWARE GEs but has two particular extensions for business collaboration: the app store and the real-time B2B collaboration core. These key components are connected with several other modules to:

- enable system integration (e.g. with loT applications).

- ensure security, privacy and trust in business collaboration.

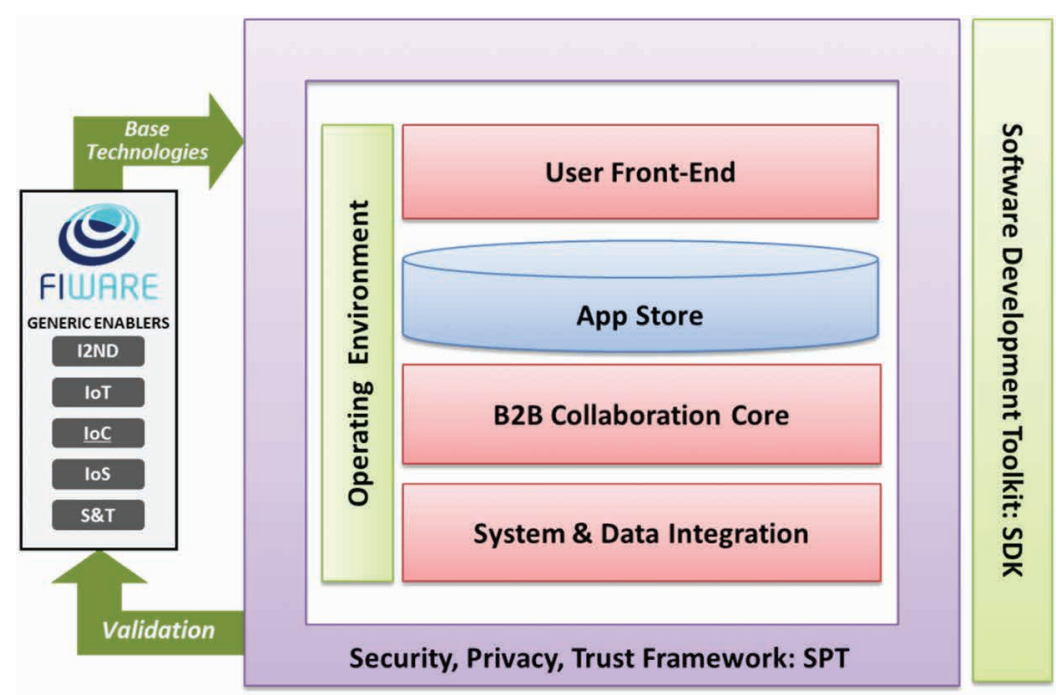

Figure 5 A high-level picture of the Flspace architecture based on FIWARE GEs. 
- provide an operating environment and software development kit to support an 'ecosystem' in which apps for the Flspace store can be developed.

The Flspace platform is accessible through various types of front-ends (e.g. web or smartphone) but also allows direct machine-to-machine (M2M) communication.

Figure 6 shows a practical example illustrating the business collaboration concept behind Flspace. It shows a use-case scenario in which a farmer gets expert advice on when to spray his crops to prevent disease, for example, spraying tomatoes in the greenhouse. The process, which involves three different stakeholders, is indicated by the white ellipses. Three apps have to work together:

- a weather information app.

- a spraying certification app.

- a spraying expert advice app.

These are provided by three independent software providers. However, by placing them within the Flspace app store, they can start to work together interoperate - as if they were one application. This is handled by the Flspace B2B collaboration engine. The Flspace platform takes care of various communication issues with external back-end systems linked by interfaces.

A working prototype of the Flspace platform and several applications, such as spraying decision support system, have been developed in the project.

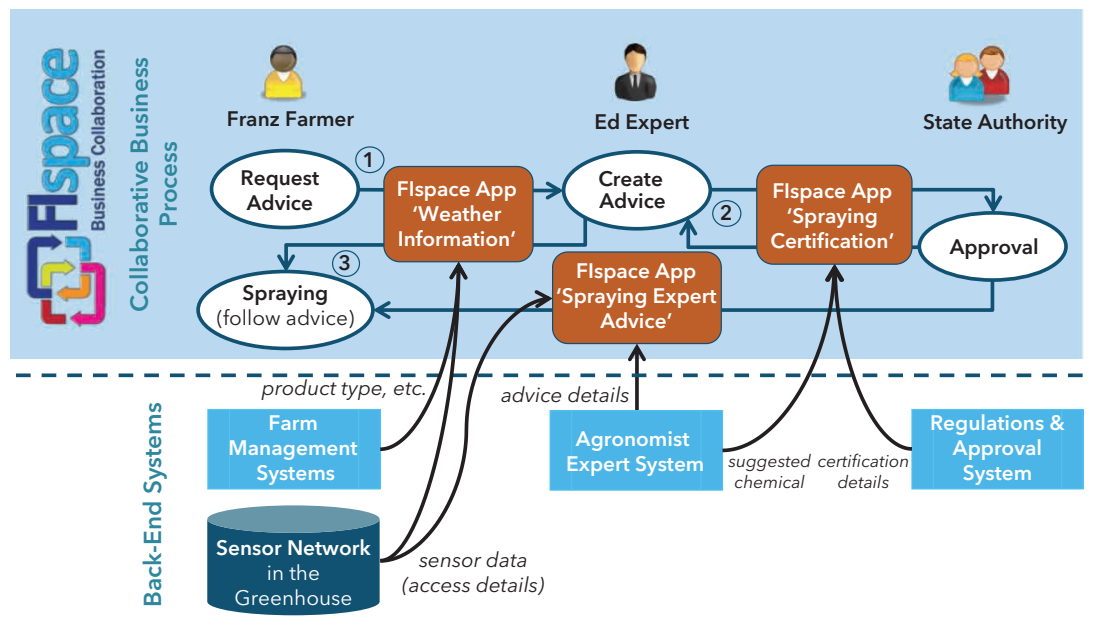

Figure $6 \mathrm{~A}$ use-case scenario illustrating the Flspace business collaboration concept. The blue rectangle represents the Flspace platform with all its functionalities that are presented in Fig. 5. Further description in the text. 
Although Flspace has not yet developed into a commercial platform, a number of elements are further being developed in commercial applications, especially within the FIWARE business ecosystem and in projects such as Internet of Food and Farm (loF2020) (Verdouw et al., 2017) (www.iof2020.eu) and SmartAgriHubs, another EU-funded project for the development of digital innovation hubs for digital transformation in agriculture (www.smartagrihubs.eu).

\section{Creating a reference architecture for interoperability, replicability and reuse}

loF2020 focusses on applications in arable crops, fruits and vegetables, dairy and meat production. Each use case is an autonomous implementation of an loT system providing a dedicated solution for a specific domain challenge. However, the project recognizes that it is important to maximize synergies across multiple use-case systems. A core concept of loF2020 is that the use-case systems function as nodes in a software ecosystem. This means ensuring the interoperability of multiple use-case systems and the reuse of IOT components across them. Figure 7 shows the architectural approach to achieve these objectives during design, development, implementation and deployment.

Developing use-case architectures will be based on a common technical reference architecture to create a shared understanding and to maximize synergies across multiple use-case systems. Each use case will use elements of the reference architecture to address its specific user requirements. The project will provide a catalogue of reusable system components which can be integrated into other loT systems to facilitate large-scale uptake. This catalogue of software units includes practical guidelines and implementation tools to facilitate uptake. The loF2020 lab will support the implementation of reusable loT components in a testbed environment. Finally, loF2020 will provide

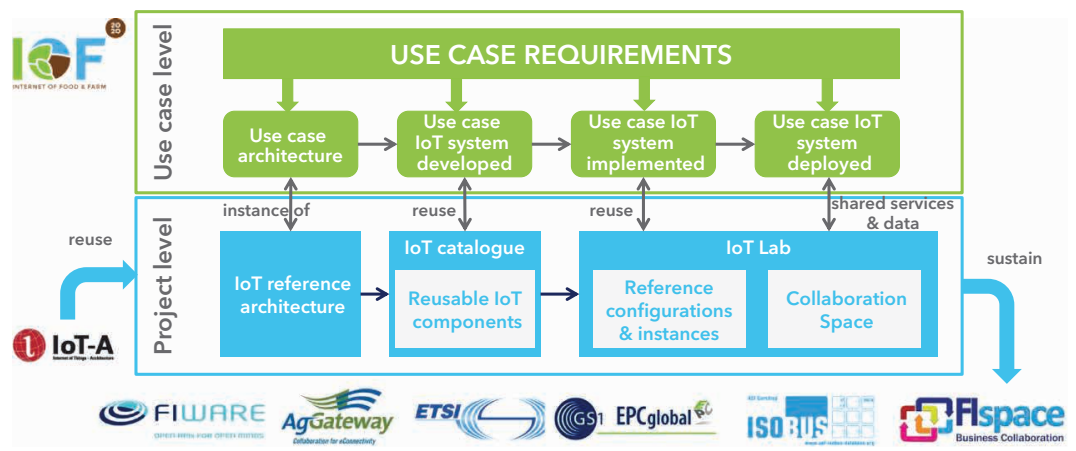

Figure 7 loF2020 architectural process ensuring re-use and interoperability of loT systems. 
a collaboration space in which services and data can be shared to facilitate interaction between individual use-case loT systems during deployment. A key feature will be reusing of components and knowledge from previous projects.

loF2020 has now developed the common reference architecture for the project, though it expects it will be constantly updated and modified in response to changing conditions (Cantera et al., 2018). The technologies and associated standards within this reference architecture address different layers that have to be tackled when developing and deploying a smart food and farming solution, as shown in Fig. 8. The main layers are:

- Physical device layer. This layer consists of loT devices and agricultural machinery deployed in the field.

- Connectivity layer. This layer enables the bidirectional transmission of data produced by devices and machinery.

- IoT service layer. Using different application-level transport protocols, this layer identifies the raw data generated from loT devices and, in some cases, actuation commands.

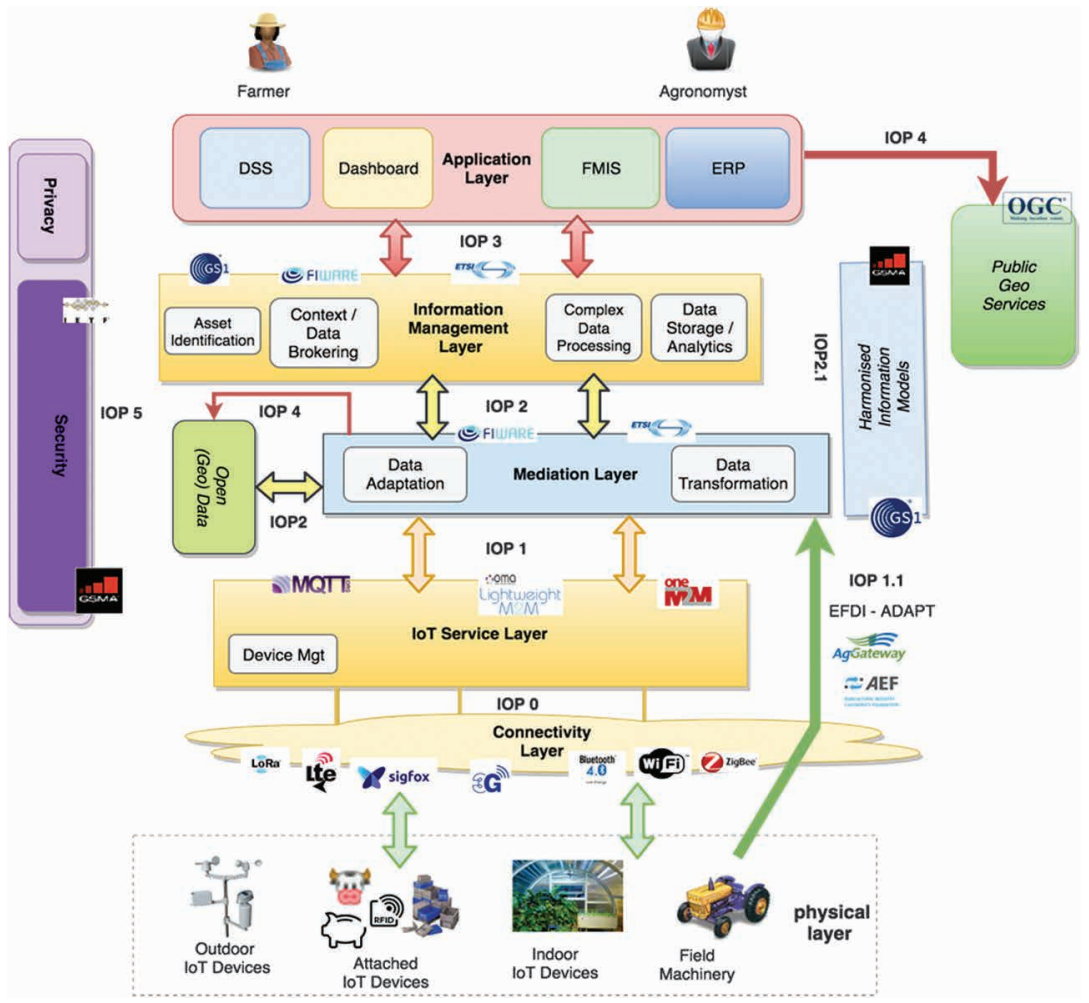

Figure 8 Overview of the loF2020 reference architecture. 
- Mediation layer. This layer transforms the raw data coming from devices or other external services into curated and harmonized data. It also aggregates some data.

- Information management layer. This layer serves mainly as a data hub. It enables the processing of all the information relevant to a food and farming solution.

- Application layer. This layer contains all the different applications such as those related to decision support (DSS), farm management (FMIS) or enterprise resource planning (ERPs).

- Security and privacy layer. This cross-cutting aims to guarantee secure access to information and devices, while protecting the data privacy of farmers and other actors in the system.

Other elements of the system include:

- Open data providers and public geo-services. Examples include public databases offering data in the agricultural domain, geo-services publishing weather or spatial data or even satellite data/image platforms.

- Harmonized information models. These define the structure and representation of the information to be managed, with a view to enable interoperability and portability of solutions in a wider ecosystem.

These interoperable elements are discussed in more detail in the following sections.

\section{Key elements in data management}

\subsection{Interoperability point 0: IoT connectivity layer}

The IoT connectivity layer enables communication between loT devices or agricultural machines (physical device layer) and data gathering platforms. It enables transmission of data from devices (uplink) and reception of actuation commands or task plans by device (downlink). There are three different enabling technologies:

- Short-range communications are based on well-established standards for wireless indoor communications based on local area networks (esp. WiFi and Bluetooth). There are also solutions for outdoor facilities as the ZigBee communication protocols (IEEE 802.15.4). There are challenges related to device battery life, network coverage and operating costs. ZigBee is being used in a solution based on wireless sensor networks for precision agriculture.

- Cellular networks allow data transmission at high speed, long range, with high reliability and autonomy. These include cellular and telco-operated 
networks exploiting 3G, 4G and 5G technologies. The main challenge is universal coverage, battery lifetime and device costs. Rural areas still have low connectivity in many areas.

- Low power wide area (LPWA) wireless technology complements existing cellular mobile network and short-range technologies, with lower costs and better consumption characteristics. LPWA networks are well suited for smart farming as they provide long-range communication and low costs for devices together with long battery life. SigFox employs a proprietary technology that enables communication using the industrial, scientific and medical (ISM) radio band. LoRa is intended for wireless battery-operated devices on a regional, national or global network. LoRaWAN targets key IoT requirements such as secure bidirectional communication, mobility and localization services. NB-loT enables a wide range of devices and services to be connected using cellular telecommunication bands and focusses specifically on indoor coverage, low cost, reliability, long battery life and the ability to connect many devices. LTE-M combines several radio access network and core network features to optimize LTE networks for loT needs and support of new category of LTE devices.

\subsection{Interoperability point 1: IoT service layer}

The IoT service layer identifies the raw data generated from loT devices through different application-level transport protocols based on different paradigms. It also offers interfaces that allow communication with devices for management or actuation purposes. The most relevant technologies enabling this interoperability are:

- MOTT is a lightweight event and message-oriented protocol allowing devices to asynchronously communicate efficiently across contained networks to remote systems. It is a publish/subscribe messaging protocol capable of delivering messages from one publisher to multiple subscribers of a topic. There are multiple MQTT open source applications and client libraries and the protocol has been successfully implemented in the smart farming domain.

- MOTT-SN can be considered a version of MQTT further adapted to wireless communication and optimized for implementation with low-cost, battery-operated devices with limited processing and storage resources. There are different open source applications of MQTT-SN.

- LWM2M is an application layer communication protocol between LWM2M sensors and LWM2M clients. It makes use of a light and compact protocol and an efficient resource data model. It is frequently used with CoAP, which 
is a specialized web-transfer protocol for use with constrained nodes and constrained networks in the loT, particularly designed for machine-tomachine applications. The OMA LWM2M protocol, which enables remote management of M2M devices, has a modern architectural REST-based architecture and is highly adaptable.

- ONEM2M is a horizontal IOT/M2M middleware platform providing common functions, that is, abstract and common APIs, for different vertical service domains. There are commercial and open source versions of this technology.

The main adoption barrier in this area is the existence of diverse standards developed by different bodies. There is a promising initiative called Web of Things, aiming to link loT devices to the web, regardless of loT protocols. Although there is no final specification yet, this technology should be developed in the future.

\subsection{Interoperability point 1.1: agricultural machinery communication layer}

This layer sits between the agricultural machinery (tractors, farm equipment etc.), and the mediation layer, and allows communication of relevant data generated from machinery to the cloud. The most relevant standards are:

- ISOBUS (ISO11783) governs electronics and data exchange between different farm machines (e.g. tractor and farm implement, for example, sprayer) and has been the de-facto standard for decades for tractor manufacturers. A local communication bus system based on CAN bus connects the tractor and other components. Data interchange and process flow are also defined in parts of this standard using ISO-XML and EFDI.

- The ADAPT framework is comprised of an agricultural application data model, a common API, and a combination of open source and proprietary data conversion plugins. Participating FMIS (farm management information system) companies are responsible for completing their own mapping of the agricultural application data model to their FMIS data model.

\subsection{Interoperability points 2, 3 and 4: mediation and information management layer}

The aim of the mediation and information management layers is to offer the right information to the right application at the right time. These layers are 
responsible for transforming raw data into information relevant and ready to be consumed by applications, so that smart behaviours are exhibited, enabling the optimization of agri-food processes.

The mediation layer, situated between the loT service layer and the information management layer (interoperability point 2), is responsible for gathering the raw data coming from devices or other external services. It then curates, harmonizes and, in some cases, aggregates the data so that it can be published as context information, or supplied to upstream data processing algorithms or analytics. In addition, this layer is also capable of sending actuation commands to the loT service layer. Finally, the mediation layer may also be used for gathering data from other data sources such as agricultural machinery or public geo-services (interoperability point 4).

The information management layer, situated between the mediation layer and the application layer, serves mainly as a data hub to enable consumption of all information relevant to an application (interoperability point 3 ). Elements include:

- FIWARE NGSI: It is a version of the OMA NGSI-9 and NGSI-10 abstract interfaces for context information management. FIWARE NGSIv2 is based on HTTP/REST and JavaScript Object Notation (JSON), following the usual de-facto industry standards. NGSI supports a powerful yet simple and well-known approach to represent context information, with a meta-model based on entities, attributes and metadata. The most popular version of FIWARE NGSI is the Orion Context Broker, which uses MongoDB as the underlying data source.

- NGSI-LD: It is an evolution of the OMA NGSI information model, designed to better support linked data, property graphs and semantics. It is being developed under the ETSI ISG CIM initiative.

- WFS: It is an interface specified by the Open GIS Consortium to allow exchange of geographic data across the web. It defines the rules for requesting and retrieving geographic information using HTTP. The interface describes the data manipulation operations for geographic features. XML-based geographic markup language is used to exchange information.

- WMS: It is a specification outlining communication mechanisms to allow disjoint software products to request and provide preassembled map imagery to a requesting client. Using WMS, a request results in a readymade map which can be displayed.

The main adoption barrier in the mediation and information management layers is the proliferation of proprietary APIs, vocabulary and incompatible data models. 


\subsection{Interoperability point 2.1: harmonized information models}

Interoperability in the agri-food sector is not only a matter of harmonized APIs (such as NGSI-LD or WFS). It also requires harmonized domain-specific data models capable of modelling different concepts relevant to different applications. Key elements include:

- CLP.26-loT big data harmonized data model, published by the GSMA IoT Programme. This is a normative document describing some harmonized data entities used in different loT domains such as agriculture. The definition of different entity types is based on JSON and the FIWARE NGSIv2 information model, while reusing some parts of schema.org.

- ADAPT framework, developed by AgGateway, includes three core elements: the ADAPT data model (ADM), a plugin manager and a set of plugins. The ADAPT framework includes a proprietary plugin which maps a system's model into the ADM model and serializes the information into a container to share it. The receiver deserializes the container using its own plugin to extract relevant information for its system.

- GS1 core business vocabulary is the data standard used within the electronic product code information service (EPCIS). It is a GS1 standard interface for capturing and sharing event data independent of any data carrier. The core business vocabulary defines elements and their values, for example, for business step identifiers, disposition identifiers, business transactions and respective types, and source/destination identifiers and types.

GS1 enables unique asset identification through different standards:

- GEPIR (Global Electronic Party Information Registry) is a unique, internetbased service that gives access to basic contact information for companies that are members of GS1. These member companies use GS1's globally unique numbering system to identify their products, physical locations or shipments.

- Global Location Number (GLN) can be used by companies to identify their locations, giving them complete flexibility to identify any type or level of location required.

- Global Trade Item Number (GTIN) can be used by a company to uniquely identify all of its trade items. GS1 defines trade items as products or services that are priced, ordered or invoiced at any point in the supply chain.

- GS1 has two GS1 keys for asset identification. The Global Returnable Asset Identifier (GRAI) is especially suitable for the management of reusable transport items, transport equipment and tools and can identify these returnable assets by type and individually for tracking and sorting purposes. 
The Global Individual Asset Identifier (GIAI) can be applied on any asset to uniquely identify and manage that asset. This could be, for example, a computer, desk, vehicle, piece of transport equipment or spare part.

- The Electronic Product Code ${ }^{\mathrm{TM}}$ (EPC) is a syntax for unique identifiers assigned to physical objects, unit loads, locations or other identifiable entities playing a role in business operations. EPCs have multiple representations, including binary forms suitable for use on radio frequency identification (RFID) tags, and text forms suitable for data sharing among the enterprise information systems (EISs). GS1's EPC tag data standard (TDS) specifies the data format of the EPC, and provides encodings for numbering schemes - including the GS1 keys - within an EPC.

\subsection{Interoperability point 5: security and privacy}

The digitalization of the agri-food industry to enhance farming processes implies that data is being generated and exchanged throughout the production process. The increasing exchange of data is a major challenge for the sector, and poses questions about privacy, data protection, intellectual property, data attribution (ownership), relationships of trust/power, storage, conservation, usability and security.

An loF2020 solution should be able to properly react to data privacy and security violations with defined procedures and should incorporate capabilities in order to secure the platform which is going to support the farming services. It needs to provide support for confidentiality, integrity, authentication, authorization, trust and non-repudiation, when needed. loF2020 has defined a set of loT Security Guidelines to be followed when implementing use cases and trials. Some relevant initiatives include:

- The EU General Data Protection Regulation (GDPR) was designed to harmonize data privacy laws across Europe, to protect and empower the data privacy of all EU citizens and to reshape the way organizations across the region approach data privacy.

- A coalition of associations from the EU agri-food chain has launched a joint EU Code of Conduct on agricultural data sharing. The Code promotes the benefits of sharing data and enables agri-business models, including agri-cooperatives and other agri-businesses, to swiftly move into digitally enhanced farming. The Code reviews contractual relations and provides guidance on the use of agricultural data, particularly the rights to access and use of data.

- GSMA loT security guidelines provide an approach to end-to-end security, including 85 detailed recommendations for secure design, development and deployment of loT services. These guidelines promote best practices 
for the secure design, development and deployment of loT services, and provide a mechanism to evaluate security measures.

- oneM2M develops technical specifications and standards which address the need for a common M2M service layer in loT. oneM2M has issued two relevant technical specifications, namely TS-0003-V2.12.1 Security Solutions and TR-0008-V2.0.1 Security.

Following these guidelines and specifications will optimize system security and protect the data rights of the different actors in the system.

\section{Conclusions}

Translating data into useful and actionable information requires appropriate software applications to be developed. This chapter has emphasized the importance of starting from a business process management perspective. Working in a supply chain network context means dealing with multiple stakeholders and their own business processes and objectives. Interoperability, the ability of computers and machines to seamlessly communicate with each other is a key challenge to address in this context. A reference architecture of various layers and interoperability points can help to create a system foundation which allows data to flow easily and effectively through the system. Moreover, it can stimulate re-use and replicability of components and use-case scenarios, leading to an increased adoption of data-driven applications in agri-food business.

\section{Where to look for further information}

The work in this chapter is largely based on a European innovation ecosystem that was developed through a series of EU-funded projects. A conceptual foundation was laid in a paper that was published in 2010 (Wolfert et al., 2010). The concepts and ideas of this paper were further elaborated and materialized through the following projects that published reports, which are detailed sources of information:

- SmartAgriFood (www.smartagrifood.eu) - Deliverable D600.2 'Plan for standardisation for large scale experimentation' contains a detailed overview of various data standards that are used in agri-food (https://c ordis.europa.eu/docs/projects/cnect/6/285326/080/deliverables/001 -D6002PlanForStandardiationFINAL2.pdf);

- Flspace (http://www.fispace.eu/) - A series of three deliverables (D500.4.x; $x=1,2,3)$ deal with various standardization issues can be found at http:// www.fispace.eu/publicdeliverables.html; 
- FIWARE (www.fiware.org) - this was the overarching project for SmartAgriFood and Flspacein the Future Internet programme that also covered various other sectors (e.g. smart cities, energy, health). FIWARE was turned into a foundation that is supported by various ICT businesses and now has a wide, active network of developers and sources, including a subcommunity on smart agrifood (https://www.fiware.org/community/s mart-agrifood/);

- IoF2020 (www.iof2020.eu) - a large-scale pilot on the application of the Internet of Things (IoT) in farming and food production. The reference architecture that is summarized in this chapter is described in more detail in several deliverables of work package three (https://www.iof2020.eu/a bout/deliverables).

In these projects, there is an active involvement of relevant standardization organizations such as:

- AgGateway Europe (https://www.aggateway.org/AboutUs/Europe.asp $x$ ) - part of the AgGateway Global Network. In this context, the ADAPT framework is of particular interest (https://www.aggateway.org/GetConne cted/ADAPT(inter-operability).aspx);

- AEF (https://www.aef-online.org/) - the Agricultural Engineering Foundation that amongst others develops and maintains the ISOBUS standard for communication between agricultural machinery;

- GS1 (www.gs1.org) - that global standards organization that provides all kinds of standards for various sectors amongst others agri-food.

\section{References}

Cantera, J. M., Issa, J. S., van der Vlugt, P., Klaeser, S., Bartram, T., Kassahun, A., Neira, I. and Milin, T. 2018. D3.3 opportunities and barriers in the present regulatory situation for system development. In: loF2020 (Ed.), loF2020 Project Deliverables.

Kaloxylos, A., Eigenmann, R., Teye, F., Politopoulou, Z., Wolfert, S., Shrank, C., Dillinger, M., Lampropoulou, I., Antoniou, E., Pesonen, L., Nicole, H., Thomas, F., Alonistioti, N. and Kormentzas, G. 2012. Farm management systems and the Future Internet era. Computers and Electronics in Agriculture 89, 130-44. doi:10.1016/j. compag.2012.09.002.

Sundmaeker, H., Verdouw, C., Wolfert, S. and Pérez Freire, L. 2016. Internet of food and farm 2020. In: Vermesan, O. and Friess, P. (Eds), Digitising the Industry - Internet of Things Connecting Physical, Digital and Virtual Worlds. River Publishers, Gistrup, Denmark and Delft, the Netherlands, pp. 129-51.

Verdouw, C. N., Wolfert, J., Beulens, A. J. M. and Rialland, A. 2016. Virtualization of food supply chains with the internet of things. Journal of Food Engineering 176, 128-36. doi:10.1016/j.jfoodeng.2015.11.009. 
Verdouw, C. N., Wolfert, S., Beers, G., Sundmaeker, H. and Chatzikostas, G. 2017. IOF2020: fostering business and software ecosystems for large-scale uptake of loT in food and farming. In: Nelson, W. (Ed.), The International Tri-Conference for Precision Agriculture in 2017, Hamilton, p. 7.

Wolfert, J., Verdouw, C. N., Verloop, C. M. and Beulens, A. J. M. 2010. Organizing information integration in agri-food - a method based on a service-oriented architecture and living lab approach. Computers and Electronics in Agriculture 70(2), 389-405. doi:10.1016/j.compag.2009.07.015.

Wolfert, J., Sørensen, C. G. and Goense, D. 2014. A future internet collaboration platform for safe and healthy food from farm to fork. 2014 Annual SRII Global Conference (SRII). IEEE, San Jose, CA, pp. 266-73. 
